\title{
DESARROLLO DE UN SISTEMA DE ADQUISICIÓN Y TRATAMIENTO DE SEÑALES ELECTROCARDIOGRÁFICAS
}

\author{
Cristian Vidal Silva $^{1} \quad$ Leopoldo Pavesi Farriol $^{2}$
}

Recibido el 7 de enero de 2004, aceptado el 23 de julio de 2004

\begin{abstract}
RESUMEN
Este trabajo es un resumen del trabajo realizado en la tesis de licenciatura en ciencias de la ingeniería de uno de los autores, el cual presenta detalles de diseño e implementación de un sistema de adquisición y procesamiento de señales electrocardiográficas. Junto con esto se señalan algunas de las problemáticas más relevantes que es posible encontrar en trabajos similares, desde el punto de vista electrónico y principalmente informático. Algunas de estas problemáticas tienen que ver con computación gráfica, diseño de algoritmos en línea y diseño de circuitos electrónicos con restricciones de tiempo real. Cabe señalar que este trabajo fue financiado por el Gobierno Regional de la VII Región del Maule, bajo la denominación de Tesis de Desarrollo Regional.
\end{abstract}

Palabras clave: ECG, electrocardiograma, complejo QRS, filtros digitales, instrumentación electrónica, electromedicina.

\section{ABSTRACT}

This contribution resumes the results obtained during the thesis work of one of the authors, and therefore some design and implementation details of both the data acquisition and the EKG signal processing modules are shown. Some of the relevant difficulties that arised during the development and which include issues related to digital algorithms design and electronics circuits are described, and the real time considerations are also mentioned.

Keywords: EKG, electrocardiogram, QRS complex, digital filters, electronic instrumentation, electromedicine.

\section{INTRODUCCIÓN}

El electrocardiograma es un registro gráfico de los potenciales eléctricos generados en el corazón durante el ciclo cardiaco [7]. El electrocardiograma suele designarse por las letras (ECG o EKG), y entrega una información muy útil acerca del funcionamiento del corazón [4]. Esta representación consiste en una línea base y varias deflexiones y ondas [2]. Colocando electrodos en diversas posiciones del cuerpo y conectando dichos electrodos a un aparato electrocardiográfico, se pueden registrar las variaciones del potencial eléctrico cardiaco [7]. La disposición específica que guardan los electrodos en el cuerpo humano recibe el nombre de "derivación" [7]. Las derivaciones más importantes reciben el nombre de Bipolares, Aumentadas y Precordiales Unipolares. En este trabajo se utilizaron sólo las derivaciones bipolares. En la Figura 1 [5] se aprecia la distribución de los electrodos, para obtener las derivaciones Bipolares, distribución conocida como triángulo de Einthoven.

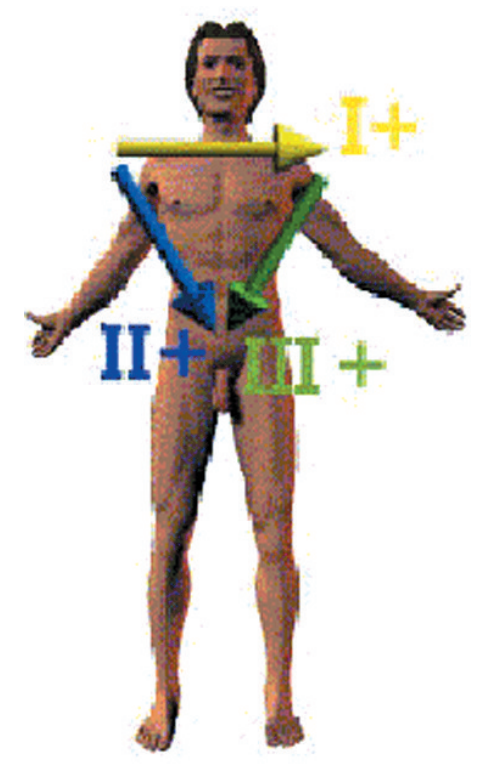

Fig. 1 Triángulo de Einthoven.

\footnotetext{
1 Est. Magister DIICC, Univ. de Concepción, Académico UCM, Chile, cristianvidal@udec.cl

2 Dpto. de Comp. e Informática, Univ. Católica del Maule, Concepción, Chile, lpavesi@spock.ucm.cl
} 


\section{ANÁLISIS DE LA SEÑAL ELECTROCARDIOGRÁFICA}

Una de las labores más importantes en un trabajo de este tipo es determinar las características de la señal a adquirir y a tratar. En este sentido hay dos enfoques equivalentes que permiten obtener información en dominios diferentes. Se denomina Dominio del Tiempo al análisis de la Señal v/s Tiempo y Dominio de la Frecuencia al análisis de las componentes frecuenciales de la señal. Esto es relevante tanto desde el punto de vista electrónico como desde el punto de vista computacional.

Respecto del Dominio del Tiempo, como se muestra en la Figura 2 [5], en todo trazo electrocardiográfico se identifican algunas formas de onda bien conocidas. Para una descripción detallada del significado de cada una de las formas de onda relevantes ver [7] o cualquier texto de electrocardiografía básica. En este trabajo sólo se considera relevante el Complejo QRS (formado por las ondas Q, R y S y cuya polaridad depende de la derivación que se esté registrando), porque precisamente se invirtió tiempo en el desarrollo de algoritmos para su detección.

Cabe señalar que un parámetro importante a ser medido en electrocardiografía es la duración del ciclo cardiaco, con lo cual es posible determinar la frecuencia cardiaca, elemento clave en el diagnóstico electrocardiográfico. Un esquema para realizar esta medición consiste en obtener el inverso multiplicativo de la duración de dos complejos QRS sucesivos.

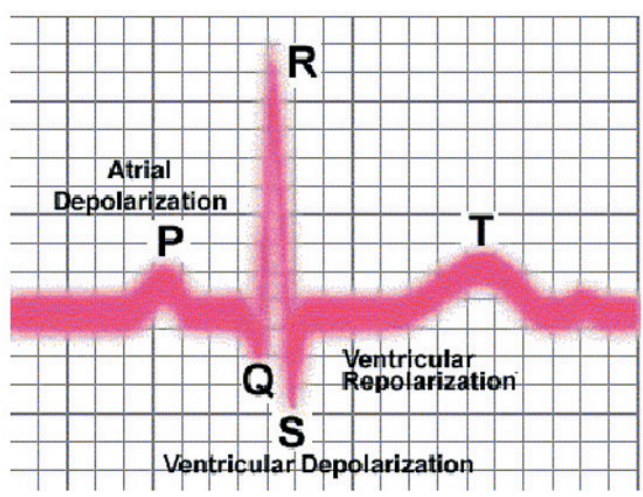

Fig. 2 Formas de onda típicas en un registro electrocardiográfico.

En términos del dominio frecuencial, se han obtenido resultados de [10] y [12] que muestran que la señal electrocardiográfica tiene un espectro frecuencial que va desde los $0 \mathrm{~Hz}$ a los $100 \mathrm{~Hz}$ como se aprecia en la siguiente tabla.

Tabla 1. Amplitud y Rango Frecuencial de algunas señales bioeléctricas típicas [10].

\begin{tabular}{|c|c|c|}
\hline Señal & $\begin{array}{c}\text { Amplitud } \\
(\mathbf{m V})\end{array}$ & $\begin{array}{c}\text { Rango } \\
\text { Frecuencial }(\mathbf{H z})\end{array}$ \\
\hline ECG & $0.02-5.0$ & $0.05-100$ \\
\hline EEG & $0.0002-0.3$ & DC -150 \\
\hline EMG & $0.1-5.0$ & DC -10000 \\
\hline
\end{tabular}

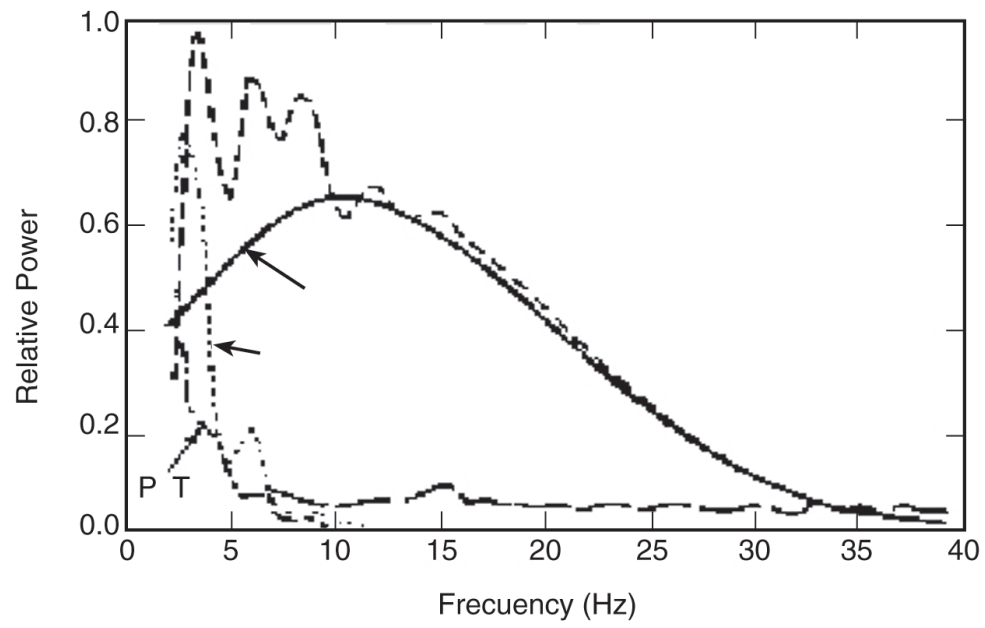

Fig. 3 Diagrama espectral de una señal ECG, complejo QRS y de algunos orígenes de ruido que afectan la señal ECG. 
Además [2], señala que el ancho de banda de la señal ECG es de $125 \mathrm{~Hz}$.

En la Figura 3 [12] se muestran espectros de frecuencias de una forma de onda específica de la señal electrocardiográfica (complejo QRS) junto a los espectros de frecuencia de varios orígenes de ruido, todos ellos comparados con el espectro frecuencial del ECG.

\section{ELECTROCARDIÓGRAFO DIGITAL}

Para realizar el diseño del circuito del electrocardiógrafo digital fueron analizados varios diseños de electrocardiógrafos presentes en la literatura. Resulta fundamental ver la construcción del diseño desde un punto de vista modular. De esta forma se pueden aislar problemáticas de manera individual. En la Figura 4 se puede apreciar un diagrama de bloques del electrocardiógrafo que se construyó en este trabajo. En [14] es posible encontrar un detalle de cada una de las partes que componen este diagrama. En lo que resta de esta sección se señalarán algunos de los problemas que se presentan en la etapa de amplificación de la señal ECG, además de mostrar los diagramas de los circuitos resultantes.

Las señales producidas por fenómenos bioeléctricos son potenciales eléctricos relativamente pequeños y requieren sofisticados amplificadores para facilitar su cuantificación [10]. El propósito principal del amplificador es incrementar el nivel de la señal que se obtiene con los electrodos excluyendo cualquier tipo de interferencia. Tal y como señala [10], la cuantificación puede realizarse usando configuraciones Unipolares o Bipolares. En el modo de cuantificación unipolar se mide la diferencia entre la señal y una tierra común. En el modo bipolar se mide la diferencia de dos orígenes de voltaje (en este caso los electrodos), respecto a una tierra común. En este caso los potenciales de interferencia generados en el punto de cuantificación aparecen en la entrada del amplificador como señales de interferencia en modo común [10]. La Figura 5 ilustra este fenómeno.

Uno de los orígenes de ruido que más interfieren en la señal ECG es la interferencia capacitiva debido al cuerpo del paciente. Este voltaje de interferencia se acopla a la señal electrocardiográfica, llegando a tener valores de $2.4 \mathrm{~V}$ aproximadamente. Este valor está muy por encima de los valores en los que fluctúa la señal electrocardiográfica $(1 \mathrm{mV}$ y $10 \mathrm{mV})$. Además de la interferencia capacitiva debido al cuerpo del paciente, es preciso señalar la interferencia capacitiva debido al equipo de medida, que se produce por la fuente de alimentación del equipo al cual llegan las señales electrocardiográficas. Otra fuente de ruido es la denominada interferencia inductiva, que es causada por la red eléctrica, la cual produce campos magnéticos que varían con el tiempo, los que a su vez inducen voltajes en la red formada por los electrodos del paciente [13].

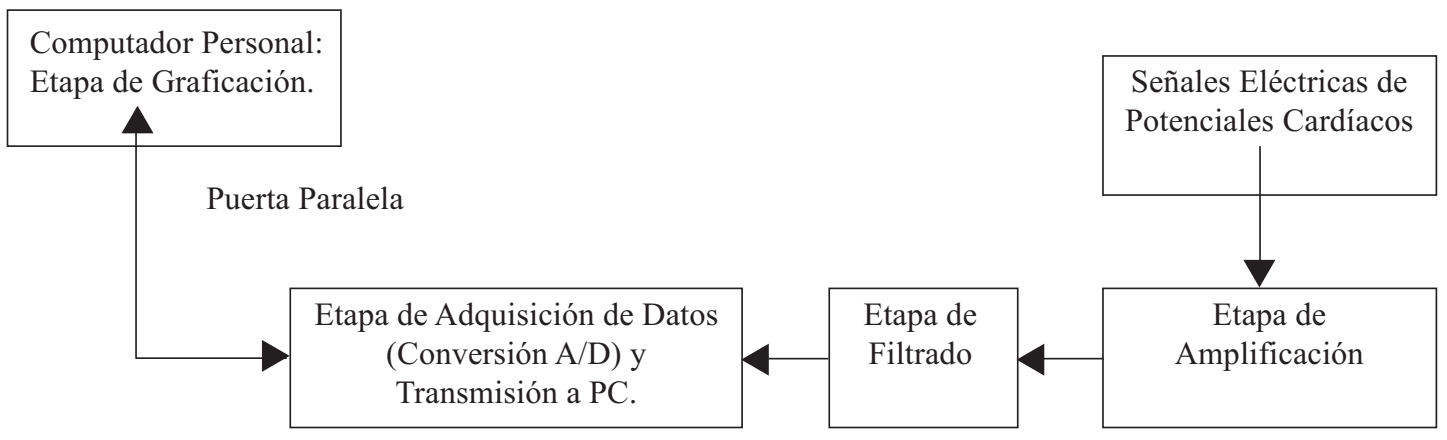

Fig. 4 Diagrama de bloques. Electrocardiógrafo digital. 


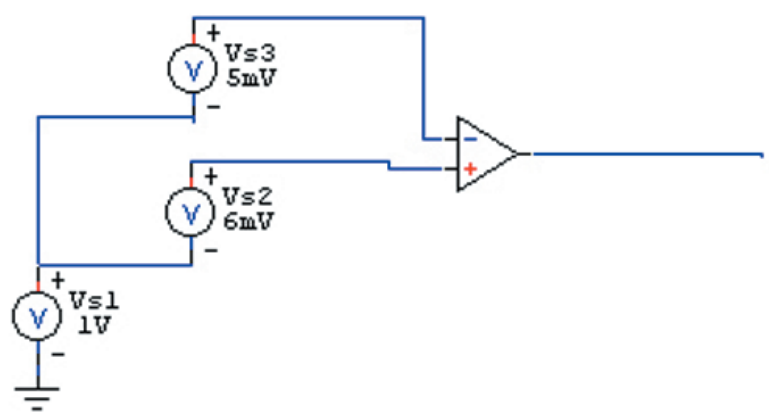

Fig. 5 Potenciales de interferencia en la cuantificación bipolar.

Por estas razones, se especifica una característica deseable de un buen amplificador que trabaje en modo diferencial, que se denomina tasa de rechazo en modo común o CMRR (common mode rejection ratio). Un problema que surge en la práctica es detallado en [13] y se denomina "desbalance en la impedancia de contacto", que se produce cuando hay diferencias en la impedancia de las interfaces entre la piel y los electrodos, de manera que el potencial en modo común será más alto en uno de los dos orígenes de voltaje. Así una fracción del voltaje en modo común será visto como voltaje diferencial y será amplificado según la ganancia del amplificador. Esto generalmente ocasiona una saturación en la siguiente etapa de amplificación (si el módulo de amplificación está compuesto por más de una etapa). Este voltaje, que generalmente es continuo, puede ser eliminado utilizando un filtro pasa alto sencillo. En conclusión, el voltaje de salida del amplificador diferencial consistirá de tres componentes [13]:

- La salida deseada debido a la amplificación diferencial de la señal electrocardiográfica.
- Una componente no deseada de la señal en modo común, debido al hecho de que la CMRR no es infinita.

- Una componente no deseada de la señal en modo común, debido al desbalance en la impedancia de contacto.

[10] señala que las señales débiles requieren por lo menos una amplificación de 1000, para producir niveles adecuados de la señal para análisis futuros. En este trabajo se utilizó el Amplificador de Instrumentación INA131, el cual tiene una CMRR fijo de 100 y el cual, en su datasheet, se señala apto para la instrumentación biomédica.

La etapa de conversión A/D se realiza una vez que la señal está amplificada. Para este propósito se utilizó el Conversor A/D TLC1541. Ambos dispositivos son de bajo costo y es posible encontrarlos en tiendas del rubro electrónico. En las Figuras 6 y 7 se ilustran los circuitos de la etapa de Amplificación y Conversión A/D. 


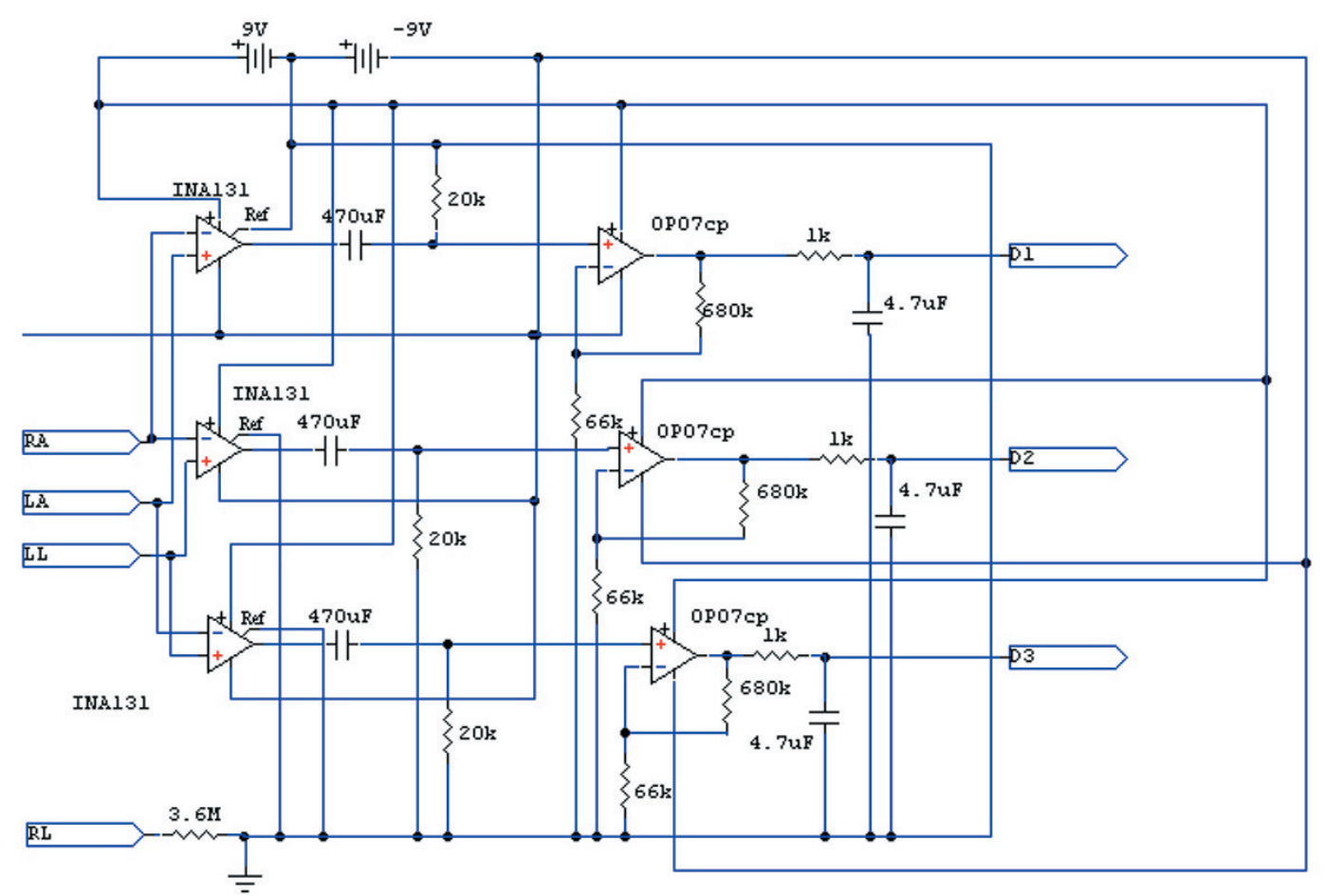

Figura 6. Circuito módulo amplificación de la señal electrocardiográfica.

La etapa de adquisición de datos tiene una componente hardware, compuesta por el Conversor A/D mismo y una componente software, que es la rutina encargada de dirigir el funcionamiento del Conversor A/D. La rutina señalada fue escrita en $\mathrm{C}$, utilizando funciones no estándares de acceso a puertos. La cantidad de muestras obtenidas es llevada a un archivo binario, el cual es rescatado más tarde por una rutina escrita en Visual Basic, para llevar a cabo el procesamiento de la señal (incluyendo la graficación, filtros y algoritmos detectores QRS).
Una vez que se han obtenido los datos de la conversión, se procede a su graficación. Este proceso es realizado "off-line" (es decir, una vez que se han obtenido las muestras deseadas) por una rutina escrita en Visual Basic, la que toma como entrada el archivo producido por la rutina de lectura del Conversor A/D. Para llevar a cabo la graficación se utilizó un objeto Picture Box (un objeto estándar dentro de Visual Basic), y para generar el desplazamiento de la ventana se utilizaron funciones de la API GDI. 


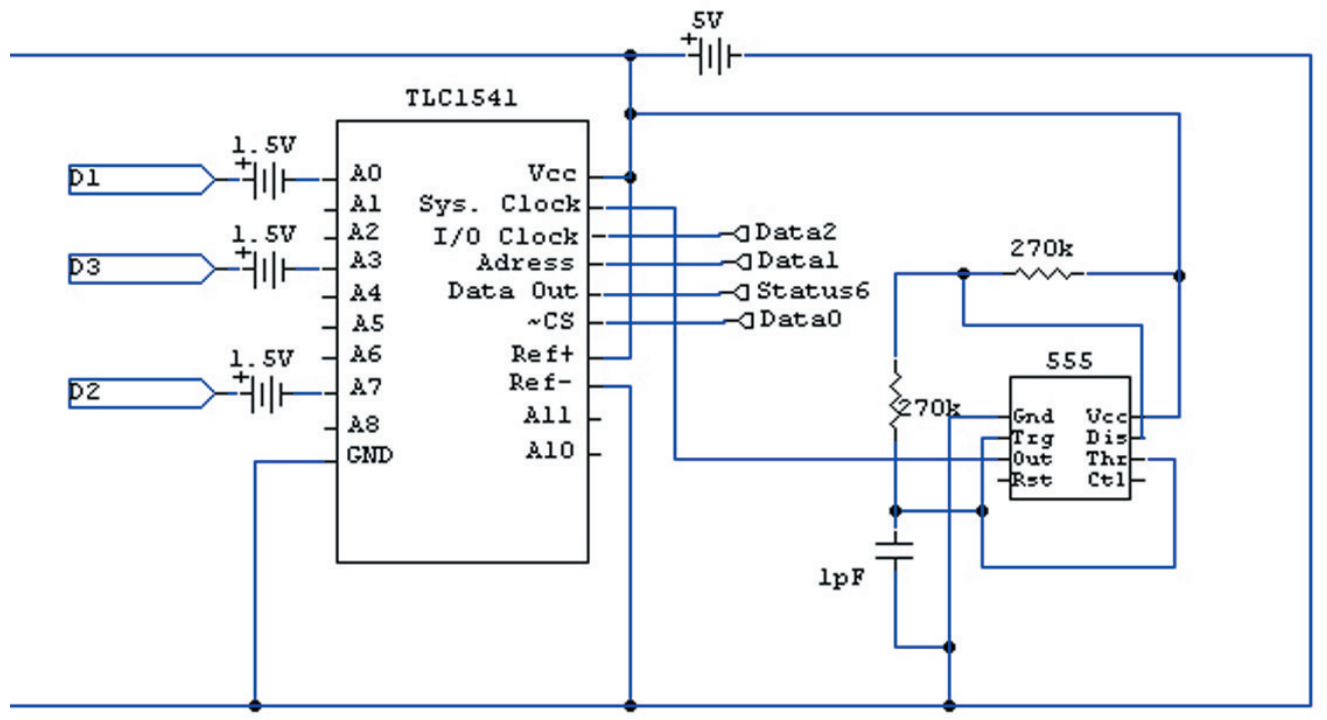

Fig. 7 Diagrama circuital del módulo de adquisición de datos.

La graficación de los resultados se realizó usando interpolación lineal simple, en una ventana que incluye 2.000 muestras (4.65 segundos) de la señal adquirida. El Teorema de Nyquist asegura que la señal puede reconstruirse usando un método de interpolación ideal (imposible de implementar en la práctica). Sin embargo, la interpolación lineal presenta buenos rendimientos para bajas frecuencias y como se apreciará más adelante permite vislumbrar con claridad una señal electrocardiográfica.

El resultado que se obtuvo de esta etapa es un Graficador Universal de Señales. En la Figura 8 se puede apreciar el rendimiento del graficador para una señal senoidal de $11 \mathrm{~Hz}$ producida por un generador de funciones.

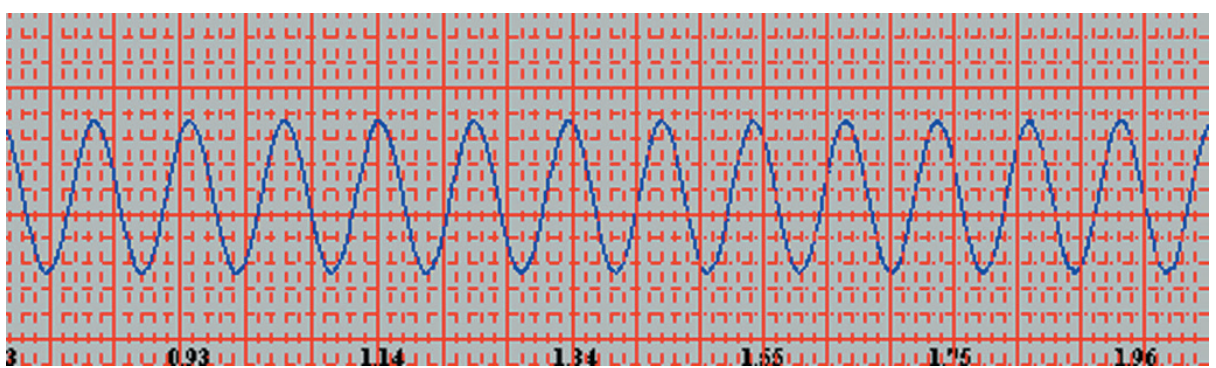

Fig. 8 Señal senoidal adquirida usando el módulo de conversión A/D.

La problemática más difícil en la rutina de graficación es lograr el efecto de ventana móvil. El objetivo es desplazar la señal cuando ésta alcanza el límite de desplazamiento, una cantidad determinada de muestras y luego dibujar en este espacio que queda libre por el desplazamiento de la imagen los siguientes datos de la señal. Esto se hace de manera cíclica, cada vez que se alcanza el límite de desplazamiento de la ventana. Esto se logró gracias a la función BitBlt de la dll gdi32 de Windows.

\section{ALGORITMOS PARA EL TRATAMIENTO DE LA SEÑAL ECG}

En este trabajo se implementaron filtros digitales con el fin de disminuir el ruido de la señal electrocardiográfica. La mayor parte de estos filtros se denominan Filtros de Lynn y son descritos en [1] y aplicados al procesamiento de señales electrocardiográficas en [9] y en [8]. Estos filtros tienen propiedades deseables de filtros en tiempo real, como lo es su fase lineal y coeficientes enteros. Los Filtros de Lynn tienen una versión Pasa Alto y Pasa Bajo. 
Dentro de la detección automática de formas de onda de la señal electrocardiográfica, la más importante de ellas es la detección del complejo QRS [2]. Esta es la característica dominante de la señal electrocardiográfica. El complejo QRS marca el inicio de la contracción del ventrículo izquierdo, por lo que la detección de este evento tiene muchas aplicaciones clínicas [11]. Por todo esto, es posible encontrar en la literatura especializada varios enfoques para la detección de este parámetro. Sin embargo, independiente del enfoque, la mayoría de los algoritmos con este propósito utilizan un prefiltrado de la señal, basado en el estudio hecho por [12] y que se mostró en la Figura 3. Al analizar esta figura se puede apreciar que una banda de paso adecuada para la eliminación de componentes indeseadas en la detección es $5 \mathrm{~Hz}-15 \mathrm{~Hz}$.
En este trabajo se implementaron dos algoritmos de detección de complejos QRS. Uno de ellos propuesto y analizado en [6]. Y otro propuesto en [9] y analizado en [8]. No se entrará en mayores detalles en estos algoritmos. Sólo señalar que ambos trabajan con técnicas clásicas de procesamiento de señales. Sin embargo, el segundo de ellos [8] es tan robusto, que aun las técnicas modernas no han logrado superar del todo su rendimiento. Para probar estos algoritmos no es necesario implementar un sistema de adquisición de datos. Existen bases de datos especializadas con cuyos registros es posible analizar el rendimiento de cualquier algoritmo detector de complejos QRS [14], donde una de las importantes es la MIT DB BIH.

En la Figura 9 se puede ver el aspecto final de la aplicación software, con un registro adquirido con el sistema de adquisición de datos.

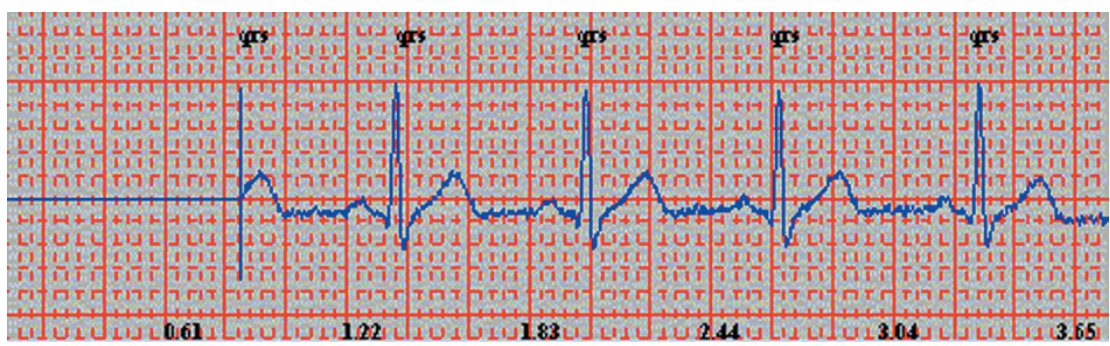

Fig. 9 Señal ECG ejemplo, adquirida con el electrocardiógrafo digital.

\section{CONCLUSIONES Y TRABAJOS FUTUROS}

Este trabajo tenía como objetivos generales la construcción de un electrocardiógrafo digital de bajo costo y el desarrollo de algoritmos para la medición de parámetros relevantes al diagnóstico médico, los cuales fueron cumplidos a cabalidad. A continuación se señalan las conclusiones de este trabajo:

- Luego de varios meses de estudio teórico y de trabajo con componentes electrónicos, se logró montar un circuito que permite la adquisición simultánea de tres derivaciones.

- La calidad del diseño que se presenta en este trabajo es de una calidad superior, en cuanto a ciertas especificaciones técnicas como el número de bits y la frecuencia de muestreo por canal, respecto a experiencias similares en la literatura.

- Tomando en cuenta sólo el costo en componentes, se puede afirmar que el diseño presentado es de bajo costo.

El sistema de adquisición de datos puede ser fácilmente extendido para la adquisición del resto de las derivaciones del electrocardiograma.
- El sistema de adquisición de datos puede ser fácilmente extendido para la adquisición de otra señal bioeléctrica.

- El módulo de graficación permite desplegar cualquier señal que se encuentre en un arreglo, con un efecto de ventana móvil, por lo que se puede afirmar que es un graficador universal de señales.

- Se analizaron e implementaron distintos tipos de filtros digitales.

- Se analizaron e implementaron dos detectores QRS de efectividad comprobada en la literatura, con lo cual es posible determinar la localización temporal de un complejo QRS.

Respecto de futuras investigaciones:

- Ampliar el uso de este equipo para la captura de otros tipos de señales bioeléctricas, tales como la señal electroencefalográfica y electromiográfica.

- Implementar un validador de algoritmos de detección QRS, basados en la MIT DB.

- Construir un algoritmo de detección de complejos QRS híbrido, con la simplicidad del algoritmo de Holsinger y con elementos del algoritmo de Pan y Tompkins. 
- Analizar otras técnicas para hacer detecciones de parámetros: Lógica Borrosa, Enfoques genéticos y Redes Neuronales.

- Hacer uso de las tecnologías de la información, tales como una base de datos, para obtener información relativa a pacientes y patologías.

Finalmente, para concluir, se debe decir que este trabajo es una buena demostración de la potencia de las aplicaciones que incluyen en su desarrollo hardware y software, sobre todo en el área de la biotecnología. La cantidad y calidad de los posibles trabajos futuros demuestran la validez de la afirmación en el aspecto académico.

\section{AGRADECIMIENTOS}

Al Dr. David Cuesta de la Universidad Politécnica de Valencia por sus valiosos aportes y excelente disposición para con los autores de este trabajo; al cardiólogo Dr. Patricio Maragaño, director del Dpto. de Cardiología del Hospital Regional de Talca, por su asesoría en el aspecto clínico y sus recomendaciones técnicas; al Dr. Carlos Cabezas, por su contribución en la fundamentación matemática de los algoritmos desarrollados; y al Gobierno Regional de la VII Región del Maule por la confianza y el patrocinio que fueron indispensables para la implementación del sistema.

\section{REFERENCIAS}

[1] M.L. Ahlstrom y otros. "Digital Filters for RealTime ECG Signal Processing Using Microprocessors", IEEE Transactions on Biomedical Engineering, vol. 32, Núm. 9, septiembre 1985, pp. 708-713.

[2] D. Cuesta. "Tesis Doctoral: Estudio de métodos para procesamiento y agrupación de señales electrocardiográficas", E.P.S. de Alcoi, septiembre 2001.

[3] E.L. Dove. "The Amplifier Operational". http://www.icaen.uiowa.edu/ bme080/ lecture_notes/lecture.html. Bioelectrical Design, The University of Iowa, Noviembre 2003.

[4] D. Dubin. "Electrocardiografía Práctica: Lesión Trazado e Interpretación”, 3 ed., McGraw Hill Interamericana, 1976
[5] H. Feldman. "A Guide to Reading and Understanding the EKG". http://endeavor.med.nyu.edu/student-org/erclub/ ekghome.html, NYU School of Medicine. 1999.

[6] G.M. Friesen y otros. "A Comparison of the Noise Sensitivity of Nine QRS Detection Algorithms", IEEE Transactions on Biomedical Engineering, vol. 31, no. 1, enero 1990, pp. 85-98.

[7] N. Dschlager. "Principios de Electrocardiografía Clínica", 7 ed., México: El Manual Moderno, 1981.

[8] P. Hamilton y otro. "Quantitative Investigation of QRS Detection Rules Using MIT/BIH Arrhythmia Database", IEEE Transactions on Biomedical Engineering, vol. 31, Núm. 3, diciembre 1986, pp. 1157-1165.

[9] Pan Jiapu y otro. “A RealTime QRS Detection Algorithm", IEEE Transactions on Biomedical Engineering, vol. 32, Núm. 3, marzo 1985, pp. 230236.

[10] Puurtinen Merja. "Bioamplifier Work". www.rgi.tut.fi/KURSSIT/7102500/biovahvistin/ biovahvistin.pdf. Tampere University of Technology, Ragnar Granit Institute, Medical Electronics Laboratory, 2003.

[11] Suppappola Seth y otros. "Nonlinear Transform of ECG Signals for Digital QRS Detection: A Quantitative Analysis", IEEE Transactions on Biomedical Engineering, vol. 41, Núm. 4, abril 1994, pp. 397-400.

[12] N. Thakor y otros. "Estimation of QRS Complex Power Spectra for Design of a QRS Filter", IEEE Transactions on Biomedical Engineering, vol. 31, no. 11 , noviembre 1984 , pp. $702-706$.

[13] N. Townsend y otros. "ECG Instrumentation". http://www.robots.ox.ac.uk/ neil/teaching/lectures/ med_elec/. Signal Processing \& Neural Networks Group, Dept. of Engineering Science, University of Oxford, 2001.

[14] C. Vidal, L. Pavesi. "Construcción de un Electrocardiógrafo Digital y Desarrollo de Algoritmos para la medición de parámetros relevantes al diagnóstico médico". Tesis de Licenciatura, Universidad Católica del Maule, enero 2004. 